\title{
Differentiation of the Peroxidase and the Oxidase Reaction by Means of the "Striatal Blood Picture."
}

Second Report of the Same Title

(6th Report of the Peroxidase Reaction).

By

TAYURU ARAKAWA and AKIRA SATO.

(荒川塯) (俈) 藤彰)

(From the Department of Pediatrics, Faculty of Medicine, Tohoku

Imperial University, Sendai. Director, Prof. A. Sato.)

The oxiduse and the peroxidase reaction of the myeloid lencocytes are very similar in appearance and are likely to lead to the assumption that both of them may be different manifestations of one and the same biological reaction. The most eloquent proof that they are essentially different reactions has been given by the "striatal blood symptom" seen in cases of the typical form of acute lethargic encephalitis and by the "striatal blood picture" "1) produced experimentally in the "peroxidase-punctured" rabbits.

Now Arakawa (one of the authors) has for some time been experimenting on a peculiar reaction in milk by use of both guaiacum tincture and one of the other tinctures used for therapentic purposes. The very first experiment was made with the tincture of digitalis. The color of the reaction was very beautiful and so distinct that such an intensity of color was never matched by any of the known peroxidase reactions of cow's milk. Besides the fact that no hydrogen peroxide had been added to the mixture the unsurpassed distinctness of the color made us doubt the true nature of the reaction, even though it was shown by Gallagher" that "alcoholic extracts of plant tissues, containing a sufficiently high content of alcohol to prevent bacterial action, are found to serve best for demonstrating this

1) Cf. K. Shoji, Tohoku J. Exp. Med., 1927, 9, 642.

2) P. H. Gallagher, Bioch. J., 1923, 17, 514. 
production of peroxide." And for convenience sake we called the reaction in question Arakawa's " tincture reaction." The name has later been used to designate such a test in which a fresh tincture of guaiacum and some one of the pharmacopeial tinctures are used (without any addition of hydrogen peroxide).

On the other hand the difference between the oxiduse and the peroxidase reaction seems clear enough, because the latter occurs only in the presence of hydrogen peroxide, while the former does not require it. But it is not so simple. If we take, for instance, the oxidase reaction of $\mathrm{W}$ in $\mathrm{k}$ ler-Schultze, the myeloid leucocytes are as is well known positive under the stain, viz. the mixture of dimethyl-p-phenylendiamin and $\alpha$-naphthol. But the same leucocytes will become positive and show exactly the same picture if hydrogen peroxide has been added to the mixture. In the former case we say that the oxidase reaction is positive, but in the latter can we simply say that now the peroxidase reaction is also pcsitive? It may indeed be very difficult to deny that in this case the peroxidase reaction has occurred, but as far as is morphologically concerned, the oxidase reaction has occurred without doubt, while no absolute proof of the cooccurrence of the peroxidase reaction can be given. However, one thing is certain ; namely, at least, it is not the peroxidase reaction alone.

Now, here is another example, perhaps a better one. K. Shoji, in our laboratory, has devised such a fundamental oxidase reagent, which, unlike the fore-runners of the kind in the hematologic literature, will not stain myeloid lencocytes at all if by itsclf alone. That was his purpose; he had subtly contrived to use the fundamental oxiduse reagent now as an oxidase reagent by help of iodine as an activator, and then as a peroxidase reaction by help of hydrogen peroxide as an activator. He however found both the oxidase and the peroxidase reaction positive on blood smears presenting the "striatal blood picture" from a successfully "peroxidasepunctured" animal, contrary to his expectation that the reaction with hydrogen peroxide would fail to be positive. And he laus come to the conclusion that an "oxidase reaction" which will become positive only through addition of hydrogen peroxide dces not necessarily mean that it is a peroxidase reaction, that this may be a (direct) oxidase reaction of a true nature under the guise of a peroxidase reaction and that the difference of both reactions will essentially be made by putting such a reaction to the test with smears presenting the "striatal blood picture." According to him an oxidase reaction which has stained the myeloid leucocytes of such smears is a (direct) oxidase reaction, whether or not hydrogen peroxide has been added. 
From the study of 'Tokuć and Arakawat) there is now no doubt that the tincture reaction is a peroxidase reaction. The fact is also presumable from Gallaghe ' $^{\text {in) }}$ study concerning plant extract. If now the assertion made by $\mathrm{Shoji}$ is correct, the tincture reaction will be negative if tried with blood smears presenting the "striatal blood picture." We tried, as shown in Table I, old tinctures of digitalis and cantharis respectively, which stained myeloid elements of normal rabbit bloods very well, with the result that each of these two tinctures failed to stain smears of the "striatal blood picture." The addition of hydrogen peroxide to the tinctures did not alter the result. It will thus follow that an oxidase reaction which becomes positive with no artificial addition of hydrogen peroxide does not necessarily mean that it is a (direct) oxiduse reaction. This may be a peroxidase reaction under the guise of an oxidase reaction. The differentiation will be essentially made if it is tested with the blood smear presenting the "striatal blood symptom" or the "striatal blood picture."

TABIL.

Reaction of myeloid leucocytes.

\begin{tabular}{|c|c|c|}
\hline Reaction & $\begin{array}{l}\text { Blood } \\
\text { smear of } \\
\text { normal } \\
\text { rabbit }\end{array}$ & $\begin{array}{l}\text { Blood smear of successfully } \\
\text { "peroxidase-punctured " rabbit; } \\
\text { (blood smear presenting the } \\
\text { " striatal blood picture") }\end{array}$ \\
\hline Oxidase reaction (W inkler-Schultze) & + & + \\
\hline Peroxidase reaction (Copper Method) & + & - \\
\hline Experiment $\int^{*}$ Fundamental $\int^{\text {a) }}$ with iodine & + & + \\
\hline K. Shoji l reagent $\quad$ (b) with $\mathrm{H}_{2} \mathrm{O}_{2}$ & + & + \\
\hline \multirow{2}{*}{ Arakawa's reaction } & + & - \\
\hline & + & - \\
\hline
\end{tabular}

* This fundamental oxidase reagent of $K$. Shoji should be so prepared that this reagent alone will not stain the leucocytes at all.

4) K. Tokué and T. Arakawa, Tohoku J. Exp. Med., 1927, 9, 650. 


\section{Conclusion.}

An apparent oxidase reaction on the myeloid leucocyte of the blood may in its true nature be a peroxidase reaction under the guise of a direct oxidase reaction. A rakawa's " tincture reaction" is apparently a direct oxidase reaction, though really a peroxidase reaction. The differentiation can be essentially shown by putting to the test a reaction in question with blood smears from a patient with the "striatal blood symptom" or those from a rabbit successfully "peroxidasc-punctured" (viz. a rabbit with the "striatal blood picture.") 